\title{
Decidable Call by Need Computations in Term Rewriting (Extended Abstract)
}

\author{
Irène Durand ${ }^{1}$ and Aart Middeldorp ${ }^{2 \star}$ \\ ${ }^{1}$ LaBRI $\quad{ }^{2}$ Institute of Information Sciences and Electronics \\ Université de Bordeaux I University of Tsukuba \\ 33405 Talence, France Tsukuba 305, Japan \\ idurand@labri.u-bordeaux.fr ami@score.is.tsukuba.ac.jp
}

\begin{abstract}
In this paper we study decidable approximations to call by need computations to normal and root-stable forms in term rewriting. We obtain uniform decidability proofs by making use of elementary tree automata techniques. Surprisingly, by avoiding complicated concepts like index and sequentiality we are able to cover much larger classes of term rewriting systems.
\end{abstract}

\section{Introduction}

The following theorem of Huet and Lévy [8] forms the basis of all results on optimal normalizing reduction strategies for orthogonal term rewriting systems (TRSs): every reducible term contains a needed redex, i.e., a redex which is contracted in every rewrite sequence to normal form, and repeated contraction of needed redexes results in a normal form, if the term under consideration has a normal form. Unfortunately, needed redexes are not computable in general. Hence, in order to obtain a computable optimal reduction strategy, we are left to find (1) decidable approximations of neededness and (2) decidable properties of TRSs which ensure that every reducible term has a needed redex identified by (1). Starting with the seminal work of Huet and Lévy [8] on strong sequentiality, these issues have been extensively investigated in the literature $[1,9,10,13,15$, 16, 19]. In all these works Huet and Lévy's notions of index, $\omega$-reduction, and sequentiality figure prominently.

We present an approach to decidable call by need computations to normal form in which issues (1) and (2) above are addressed directly. Besides facilitating understanding this enables us to cover much larger classes of TRSs. For instance, an easy consequence of our work is that every orthogonal right-ground TRS admits a computable call by need strategy whereas none of the sequentialitybased approaches cover all such TRSs. Our approach is based on the easy but

\footnotetext{
* The work reported in this paper was initiated in March 1995 when the second author was staying at the University of Bordeaux. The support of LaBRI and the hospitality of the "Programmation Symbolique" group headed by Robert Strandh are gratefully acknowledged.
} 
fundamental observation that needed redexes are uniform but not independent of other redexes in the same term. Uniformity means that only the position of a redex in a term counts for determining neededness.

Inspired by Comon [1], our decidability results heavily rely on tree automata techniques. However, by assigning a greater role to ground tree transducers we don't need to rely on the (difficult) connection between weak second-order monadic logic and tree automata, even when considering the shallow and growing approximations of Comon and Jacquemard [1,9].

In this paper we also study decidable call by need computations to rootstable form, the underlying theory of which is expounded in Middeldorp [14]. This is more complicated because root-needed redexes, which are redexes that are contracted in every rewrite sequence to root-stable form, are not uniform.

The remainder of this paper is organized as follows. In the next section we recall the necessary background of term rewriting and tree automata. In Section 3 we present sufficient conditions for neededness in terms of approximations. Growing and shallow approximations are introduced in Section 4. In Section 5 we present our framework for decidable call by need computations to normal form. We also include a comparison with the sequentiality-based approach. Decidable approximations of root-neededness is the topic of Section 6 and in Section 7 we consider decidable call by need computations to root-stable form. Due to lack of space, we concentrate on decidability issues in these last two sections. Results on the relationships between the various classes of TRSs have been omitted. For the same reason we don't address the important issue of complexity in this paper. The main purpose of this paper is to get our framework to decidable call by need computations across.

\section{Preliminaries}

We assume familiarity with the basic notions of term rewriting (see e.g. $[5,12]$ ). A rewrite rule $l \rightarrow r$ is a pair of terms such that the left-hand side $l$ is not a variable. We find it convenient not to impose the usual restriction that variables which occur in the right-hand side $r$ occur in $l$ as well. A TRS over a signature $\mathcal{F}$ is a set $\mathcal{R}$ of rewrite rules between terms in $\mathcal{T}(\mathcal{F}, \mathcal{V})$. Here $\mathcal{V}$ is a countably infinite set of variables. A ground term doesn't contain variables. A linear term doesn't contain multiple occurrences of the same variable. A redex is an instance of the left-hand side of a rewrite rule. The set of all ground redexes of a TRS $\mathcal{R}$ is denoted by REDEX $\mathcal{R}_{\mathcal{R}}$. A normal form is a term without redexes. The set of all ground normal forms of $\mathcal{R}$ is denoted by $\mathrm{NF}_{\mathcal{R}}$. A term is root-stable if it cannot be rewritten to a redex. The set of all root-stable ground terms of $\mathcal{R}$ is denoted by $\mathrm{RS}_{\mathcal{R}}$. A TRS is left-linear (right-linear, linear) if the left-hand sides (right-hand sides, both left and right-hand sides) of its rewrite rules are linear terms. A TRS is right-ground (ground) if the right-hand sides (left and righthand sides) of its rewrite rules are ground terms. A binary relation $R$ on terms is called parallel if $C\left[s_{1}, \ldots, s_{n}\right] R C\left[t_{1}, \ldots, t_{n}\right]$ whenever $s_{1} R t_{1}, \ldots, s_{n} R t_{n}$, for all contexts $C$ and terms $s_{1}, \ldots, s_{n}, t_{1}, \ldots, t_{n}$. The parallel closure $R^{\|}$of a 
binary relation $R$ on terms is the smallest parallel relation that contains $R$. The parallel closure of the rewrite relation $\rightarrow_{\mathcal{R}}$ of a TRS $\mathcal{R}$ is denoted by $\|_{\mathcal{R}}$ rather than $\rightarrow_{\mathcal{R}}^{\|}$.

A redex $\Delta$ in a term $t$ is needed if in every rewrite sequence from $t$ to normal form a descendant of $\Delta$ is contracted. Let $A: s \rightarrow^{*} t$ be a rewrite sequence and $\Delta$ a redex in $s$. We write $\Delta \perp A$ if no descendant of $\Delta$ is contracted in $A$. So a redex $\Delta$ in a term $s$ is needed if and only if $t$ is not a normal form whenever $A: s \rightarrow^{*} t$ with $\Delta \perp A$. Lemma 8 below gives an equivalent definition of neededness that doesn't depend on the notion of descendant.

\section{Orthogonality}

A left-linear TRS without critical pairs and whose rewrite rules satisfy the additional restriction that variables which occur in the right-hand side occur also in the left-hand side is called orthogonal. The following theorem of Huet and Lévy [8] forms the basis of all results on optimal normalizing reduction strategies for orthogonal TRSs.

Theorem 1. Let $\mathcal{R}$ be an orthogonal TRS. Every reducible term contains a needed redex and repeated contraction of needed redexes results in a normal form, if the term under consideration has a normal form.

Middeldorp [14] generalized the above result to rewrite sequences to rootstable form. A redex $\Delta$ in a term $t$ is root-needed if in every rewrite sequence from $t$ to root-stable form a descendant of $\Delta$ is contracted. In [14] it is argued that root-neededness is the proper generalization of neededness when it comes to optimal infinitary normalization.

Theorem 2. Let $\mathcal{R}$ be an orthogonal TRS. Every non-root-stable term contains a root-needed redex and repeated contraction of root-needed redexes results in a root-stable form, if the term under consideration has a root-stable reduct.

\section{Tree Automata}

A tree automaton is a quadruple $\mathcal{A}=\left(\mathcal{F}, Q, Q_{f}, \Delta\right)$ consisting of a finite signature $\mathcal{F}$, a finite set $Q$ of states, disjoint from $\mathcal{F}$, a subset $Q_{f} \subseteq Q$ of final states, and a set of transition rules $\Delta$. Every transition rule has one of the following two forms: $f\left(q_{1}, \ldots, q_{n}\right) \rightarrow q$ with $f \in \mathcal{F}$ and $q_{1}, \ldots, q_{n}, q \in Q$, or $q \rightarrow q^{\prime}$ with $q, q^{\prime} \in Q$. Transition rules of the second form are called $\epsilon$-rules. Note that a tree automaton $\mathcal{A}=\left(\mathcal{F}, Q, Q_{f}, \Delta\right)$ is simply a finite ground TRS $(\mathcal{F} \cup Q, \Delta)$ whose rewrite rules have a special shape, together with a subset $Q_{f}$ of $Q$. The induced rewrite relation on $\mathcal{T}(\mathcal{F} \cup Q)$ will be denoted by $\rightarrow_{\mathcal{A}}$. We say that a term $t \in \mathcal{T}(\mathcal{F})$ is accepted by $\mathcal{A}$ if $t \rightarrow{ }_{\mathcal{A}}^{*} q$ for some $q \in Q_{f}$. The set of all such terms is denoted by $L(\mathcal{A})$. A subset $L \subseteq \mathcal{T}(\mathcal{F})$ is called recognizable if there exists a tree automaton $\mathcal{A}=\left(\mathcal{F}, Q, Q_{f}, \Delta\right)$ such that $L=L(\mathcal{A})$. It is well-known that every recognizable language is accepted by a deterministic tree automaton. A deterministic automaton has neither $\epsilon$-rules nor different rules with the same left-hand side. In this paper we make use of the following properties. 
Lemma 3. The set $\mathcal{T}(\mathcal{F})$ is recognizable. The intersection $L_{1} \cap L_{2}$ and difference $L_{1} \backslash L_{2}$ of recognizable subsets $L_{1}, L_{2} \subseteq \mathcal{T}(\mathcal{F})$ are recognizable. The complement $L^{c}$ of a recognizable subset $L \subseteq \mathcal{T}(\mathcal{F})$ is recognizable.

Lemma 4. Let $L \subseteq \mathcal{T}(\mathcal{F})$ be recognizable. Given a term $t \in \mathcal{T}(\mathcal{F})$, it is decidable whether $t \in L$. It is decidable whether $L$ is empty.

The following well-known result connects tree automata with TRSs.

Lemma 5. Let $\mathcal{R}$ be a finite left-linear TRS. The sets $\mathrm{REDEX}_{\mathcal{R}}$ and $\mathrm{NF}_{\mathcal{R}}$ are recognizable.

A ground tree transducer (GTT for short) is a pair $\mathcal{G}=(\mathcal{A}, \mathcal{B})$ of tree automata over the same signature $\mathcal{F}$. Let $s, t \in \mathcal{T}(\mathcal{F})$. We say that the pair $(s, t)$ is accepted by $\mathcal{G}$ if $s \rightarrow_{\mathcal{A}}^{*} \cdot{ }_{\mathcal{B}}^{*} \leftarrow t$. The set of all such pairs is denoted by $L(\mathcal{G})$. Observe that $L(\mathcal{G})$ is a binary relation on $\mathcal{T}(\mathcal{F})$. A binary relation on ground terms is called recognizable if there exists a GTT that accepts it. Every recognizable relation is parallel. GTTs were introduced by Dauchet and Tison [4] in order to prove that confluence is a decidable property of ground TRSs. In this paper we make use of the following closure properties. The last one is from [2].

Lemma 6. Let $R$ be a recognizable relation on $\mathcal{T}(\mathcal{F})$. The inverse $R^{-1}$ and transitive closure $R^{+}$of $R$ are recognizable. If $L$ is a recognizable subset of $\mathcal{T}(\mathcal{F})$ then $R[L]=\{s \mid s R$ for some $t \in L\}$ is recognizable.

In the literature $R[L]$ often denotes the different set $\{t \mid s R t$ for some $s \in L\}$. We find our choice more convenient.

We would like to emphasize that there are many other notions of recognizability in the literature besides the two introduced in this subsection. In this paper a subset of terms is recognizable if it is accepted by a tree automaton and a binary relation on terms is recognizable if it is accepted by a GTT. (In [3] recognizable relations are called GTT-relations.)

\section{Weak Second-Order Monadic Logic}

The material in this subsection is only used in Section 7. We assume familiarity with $\mathrm{WS} k \mathrm{~S}$, the weak second-order monadic logic with $k$ successors. See Thomas [18, Section 11] for a discussion of WS2S.

Let $\mathcal{F}$ be a finite signature. Let $k$ be the maximal arity of function symbols in $\mathcal{F}$ and let $n$ be the cardinality of $\mathcal{F}$. A term $t \in \mathcal{T}(\mathcal{F})$ is represented in WSkS using $n+1$ set variables $X$ and $X_{f}$ for every $f \in \mathcal{F}$. In the following we write $\mathbf{X}$ for the sequence $X, X_{f}$ for $f \in \mathcal{F}$. The WSkS formula term $(\mathbf{X})$ :

$$
\begin{aligned}
& X=\bigcup_{f \in \mathcal{F}} X_{f} \wedge \bigwedge_{f \in \mathcal{F}} \forall x \in X_{f}\left[\bigwedge_{i=1}^{\operatorname{arity}(f)} x \cdot i \in X \wedge \bigwedge_{i=\operatorname{arity}(f)+1}^{k} x \cdot i \notin X\right] \wedge \\
& \bigwedge_{f \neq g \in \mathcal{F}} X_{f} \cap X_{g}=\varnothing \wedge \forall x \in X \forall y<x[y \in X]
\end{aligned}
$$


expresses that $\mathbf{X}$ encodes a term in $\mathcal{T}(\mathcal{F})$. If term $(\mathbf{T})$ holds for the sequence $\mathbf{T}$ of sets of positions then we define $t_{\mathbf{T}}$ to be the term in $\mathcal{T}(\mathcal{F})$ uniquely determined by $\operatorname{Pos}(t)=T$ and $\operatorname{root}\left(t_{\mid p}\right)=f$ if $p \in T_{f}$, for all $p \in T$. A subset $L$ of $\mathcal{T}(\mathcal{F})$ is called WSkS definable if there exists a WSkS formula $\phi$ with free variables $\mathbf{X}$ such that $L=\left\{t_{\mathbf{T}} \mid \operatorname{term}(\mathbf{T}) \wedge \phi(\mathbf{T})\right\}$.

Theorem 7 (Doner [6], Thatcher and Wright [17]). A set $L \subseteq \mathcal{T}(\mathcal{F})$ is WSkS definable if and only if it is recognizable.

\section{Decidable Approximations of Neededness}

In the remaining part of the paper we are dealing with finite TRSs only. Moreover, we consider rewriting on ground terms only. So we assume that the set of ground terms is non-empty. This requirement entails no loss of generality. It is undecidable whether a redex in a term is needed with respect to a given (orthogonal) TRS. In this section we present decidable sufficient conditions for a redex to be needed.

Let $\mathcal{R}$ be a TRS over a signature $\mathcal{F}$. Throughout this and the next two sections we assume the existence of a constant $\bullet$ not appearing in $\mathcal{F}$ and we view $\mathcal{R}$ as a TRS over the extended signature $\mathcal{F}_{\bullet}=\mathcal{F} \cup\{\bullet\}$. So NF $\mathcal{R}_{\mathcal{R}}$ consists of all terms in $\mathcal{T}\left(\mathcal{F}_{\bullet}\right)$ that are in normal form. Let $\mathcal{R} \bullet$ be the TRS $\mathcal{R} \cup\{\bullet \rightarrow \bullet\}$. Note that $\mathrm{NF}_{\mathcal{R}}$. coincides with $\mathrm{NF}_{\mathcal{R}} \cap \mathcal{T}(\mathcal{F})$. The following easy lemma gives an alternative definition of neededness, not depending on the notion of descendant.

Lemma 8. Let $\mathcal{R}$ be an orthogonal TRS over a signature $\mathcal{F}$. Redex $\Delta$ in term $C[\Delta] \in \mathcal{T}(\mathcal{F})$ is needed if and only if there is no term $t \in \mathrm{NF}_{\mathcal{R}}$. such that $C[\bullet] \rightarrow_{\mathcal{R}}^{*} t$.

Proof. Suppose there is a term $t \in \mathrm{NF}_{\mathcal{R}}$. such that $C[\bullet]_{\mathcal{R}}^{*} t$. Replacing every occurrence of $\bullet$ by $\Delta$ yields a sequence $A: C[\Delta] \rightarrow_{\mathcal{R}}^{*} t$ with $\Delta \perp A$. Hence $\Delta$ is not needed. Conversely, if $\Delta$ is not needed then there exists a rewrite sequence $A: C[\Delta] \rightarrow_{\mathcal{R}}^{*} t$ with $t \in \mathrm{NF}_{\mathcal{R}}$ and $\Delta \perp A$. Replacing every descendant of $\Delta$ in $A$ by $\bullet$ yields a sequence $C\left[\bullet \rightarrow_{\mathcal{R}}^{*} t\right.$. (Here we use orthogonality. Note that because $t$ is a normal form there are no descendants of $\Delta$ in $t$ left.)

Note that Lemma 8 implies that needed redexes are uniform, which means that only the position of a redex in a term is important for determining neededness. So if redex $\Delta$ in term $C[\Delta]$ is needed then so is redex $\Delta^{\prime}$ in $C\left[\Delta^{\prime}\right]$.

Because the relation $\rightarrow_{\mathcal{R}}^{*}$ is in general not computable, neededness is undecidable. In order to obtain decidable sufficient conditions, the idea is now to approximate $\rightarrow_{\mathcal{R}}^{*}$ by $\rightarrow_{\mathcal{S}}^{*}$ for some suitable TRS $\mathcal{S}$ such that it is decidable whether a term has an $\mathcal{S}$-reduct in $\mathrm{NF}_{\mathcal{R}}$.

Definition 9. Let $\mathcal{R}$ and $\mathcal{S}$ be TRSs over the same signature. We say that $\mathcal{S}$ approximates $\mathcal{R}$ if $\rightarrow_{\mathcal{R}}^{*} \subseteq \rightarrow_{\mathcal{S}}^{*}$ and $\mathrm{NF}_{\mathcal{R}}=\mathrm{NF}_{\mathcal{S}}$. 
Definition 10. Let $\mathcal{R}$ be a TRS over a signature $\mathcal{F}$. We say that redex $\Delta$ in $C[\Delta] \in \mathcal{T}(\mathcal{F})$ is $\mathcal{R}$-needed if there is no term $t \in \mathrm{NF}_{\mathcal{R}}$. such that $C\left[\bullet \rightarrow_{\mathcal{R}}^{*} t\right.$.

So for every orthogonal TRS $\mathcal{R}$, a redex is needed if and only if it is $\mathcal{R}$-needed. The following lemma is immediate from the definitions.

Lemma 11. Let $\mathcal{R}$ be a TRS and let $\mathcal{S}$ be an approximation of $\mathcal{R}$. Every $\mathcal{S}$ needed redex is $\mathcal{R}$-needed.

In the literature four approximations have been studied. These approximations have the same left-hand sides as the original TRS $\mathcal{R}$, hence the second requirement in the definition of approximation is trivially satisfied, but differ in the way they treat the right-hand sides of the rewrite rules of $\mathcal{R}$. In this section we introduce the two simplest approximations. The other two are dealt with in the next section.

Definition 12. Let $\mathcal{R}$ be a TRS. The TRS $\mathcal{R}_{\mathrm{s}}$ is obtained from $\mathcal{R}$ by replacing the right-hand side of every rewrite rule by a variable that does not occur in the corresponding left-hand side.

The idea of approximating a TRS by ignoring the right-hand sides of its rewrite rules is due to Huet and Lévy [8]. Our $\mathcal{R}_{\mathrm{s}}$-needed redexes coincide with their strongly needed redexes. A better approximation is obtained by preserving the non- $v$ ariable parts of the right-hand sides of the rewrite rules.

Definition 13. Let $\mathcal{R}$ be a TRS. The TRS $\mathcal{R}_{\mathrm{nv}}$ is obtained from $\mathcal{R}$ by replacing the variables in the right-hand sides of the rewrite rules by pairwise distinct variables that do not occur in the corresponding left-hand sides.

The idea of approximating a TRS by ignoring the variables in the righthand sides of the rewrite rules is due to Oyamaguchi [16]. Note that $\mathcal{R}_{\mathrm{nv}}=\mathcal{R}$ whenever $\mathcal{R}$ is right-ground. Hence for every orthogonal right-ground TRS $\mathcal{R}$, a redex is needed if and only if it is $\mathcal{R}_{\mathrm{nv}}$-needed.

In the remainder of this section we show that it is decidable whether a redex in a term is $\mathcal{S}$-needed for every left-linear TRS $\mathcal{R}$ and $\mathcal{S}=\mathcal{R}_{\mathrm{s}}$ or $\mathcal{S}=\mathcal{R}_{\mathrm{nv}}$.

Definition 14. Let $\mathcal{R}$ be a TRS over a signature $\mathcal{F}$. The set of all terms $C[\bullet] \in \mathrm{M}_{\bullet}$ such that there is no term $t \in \mathrm{NF}_{\mathcal{R}}$. with $C\left[\bullet \rightarrow_{\mathcal{R}}^{*} t\right.$ is denoted by $\mathcal{R}$-NEEDED. Here $M_{\bullet}$ consists of all terms in $\mathcal{T}\left(\mathcal{F}_{\bullet}\right)$ that contain exactly one occurrence of $\bullet$.

So redex $\Delta$ in $C[\Delta] \in \mathcal{T}(\mathcal{F})$ is $\mathcal{R}$-needed if and only if $C[\bullet] \in \mathcal{R}$-NEEDED. The following result presents a sufficient condition for the decidability of $\mathcal{R}$ neededness.

Theorem 15. Let $\mathcal{R}$ be a TRS. If $\left(\rightarrow_{\mathcal{R}}^{*}\right)\left[\mathrm{NF}_{\mathcal{R}}\right.$.] is recognizable then $\mathcal{R}$-NEEDED is recognizable. 
Proof. We have $\mathcal{R}$-NEEDED $=\left(\rightarrow_{\mathcal{R}}^{*}\right)\left[\mathrm{NF}_{\mathcal{R}_{\bullet}}\right]^{c} \cap \mathrm{M}_{\bullet}$. The set $\mathrm{M}_{\bullet}$ is easily shown to be recognizable. Hence the result follows from Lemma 3.

Next we show that for the two approximations defined above, the premise of the above theorem is satisfied, hence $\mathcal{R}_{\mathrm{s}}$-neededness and $\mathcal{R}_{\mathrm{nv}}$-neededness are decidable.

Lemma 16. Let $\mathcal{R}$ be a left-linear TRS. The relations $\rightarrow_{\mathcal{R}_{\mathrm{s}}}^{*}$ and $\rightarrow_{\mathcal{R}_{\mathrm{nv}}}^{*}$ are recognizable.

Proof. Because recognizable relations are closed under transitive closure and $H^{+}=\rightarrow^{*}$, it suffices to show that $\|_{\mathcal{R}_{\mathrm{s}}}$ and $\|_{\mathcal{R}_{\mathrm{nv}}}$ are recognizable. First we show the recognizability of $\|_{\mathcal{R}_{\mathrm{nv}}}$. Let $\mathcal{R}_{\mathrm{nv}}=\left\{l_{i} \rightarrow r_{i} \mid 1 \leqslant i \leqslant n\right\}$. Define the GTT $\mathcal{G}_{\text {nv }}$ as the pair of tree automata $\mathcal{A}$ and $\mathcal{B}$ that accept in state $\mathrm{i}$ all instances of $l_{i}$ and $r_{i}$, respectively. Moreover, we may assume that the two tree automata share no other states. Hence $L\left(\mathcal{G}_{\mathrm{nv}}\right)=\|_{\mathcal{R}_{\mathrm{nv}}}$. The recognizability of $\|_{\mathcal{R}_{\mathrm{s}}}$ is obtained by replacing $\mathcal{B}$ by the tree automaton $\mathcal{C}$ that accepts in state $\mathrm{i}$ all terms.

Table 1 shows the tree automata $\mathcal{A}, \mathcal{B}$, and $\mathcal{C}$ used in the proof of the above lemma for the following TRS $\mathcal{R}$ :

$$
\begin{aligned}
1: & f(g(x), a) & \rightarrow f(h(h(x)), x) \\
2: & h(a) & \rightarrow h(b) \\
3: & h(f(x, b)) & \rightarrow x
\end{aligned}
$$

The underlinings are to ensure that only states 1,2 , and 3 are shared between $\mathcal{A}$ and $\mathcal{B}(\mathcal{C})$. Consider the tree automaton $\mathcal{A}$. Its states are $*, \mathrm{a}, \mathrm{b}, \mathrm{g}(*)$, and $\mathrm{f}(*, \mathrm{~b})$. In state $*$ all ground terms are accepted. The purpose of the second group of transition rules is to recognize all ground instances of proper non-variable subterms of the left-hand sides of $\mathcal{R}$. So in state a only the term $a$ is accepted, whereas in state $\mathrm{f}(*, \mathrm{~b})$ all ground terms of the form $f(t, b)$ are accepted. The third group of transition rules corresponds to the left-hand sides of $\mathcal{R}$.

Lemma 17. Let $\mathcal{R}$ be a left-linear TRS. The set $\left(\rightarrow_{\mathcal{S}}^{*}\right)\left[\mathrm{NF}_{\mathcal{S}_{\bullet}}\right]$ is recognizable for $\mathcal{S}=\mathcal{R}_{\mathrm{s}}$ and $\mathcal{S}=\mathcal{R}_{\mathrm{nv}}$.

Proof. Since $\mathrm{NF}_{\mathcal{S}_{\bullet}}=\mathrm{NF}_{\mathcal{R}_{\bullet}}$ is recognizable by Lemma 5 , the recognizability of $\left(\rightarrow{ }_{\mathcal{S}}^{*}\right)\left[\mathrm{NF}_{\mathcal{S}_{\bullet}}\right]$ is a consequence of Lemmata 6 and 16 .

Hence the following corollary is a consequence of the first part of Lemma 4 .

Corollary 18. Let $\mathcal{R}$ be a left-linear TRS over a signature $\mathcal{F}$. It is decidable whether a redex in a term in $\mathcal{T}(\mathcal{F})$ is $\mathcal{S}$-needed for $\mathcal{S}=\mathcal{R}_{\mathrm{s}}$ and $\mathcal{S}=\mathcal{R}_{\mathrm{nv}}$.

In particular, needed redexes are computable in orthogonal right-ground TRSs. 
Table 1. The tree automata $\mathcal{A}, \mathcal{B}$, and $\mathcal{C}$.

\begin{tabular}{|c|c|c|}
\hline$a \rightarrow *$ & $a \rightarrow *$ & $a \rightarrow *$ \\
\hline$b \rightarrow *$ & $b \rightarrow \bar{*}$ & $b \rightarrow \bar{*}$ \\
\hline$f(*, *) \rightarrow *$ & $f(*, *) \rightarrow \bar{*}$ & $f(*, *) \rightarrow *$ \\
\hline$g(*) \rightarrow *$ & $\bar{g}(\bar{*}) \rightarrow \bar{*}$ & $\bar{g}(\bar{*}) \rightarrow \bar{*}$ \\
\hline$h(*) \rightarrow *$ & $h(\bar{*}) \rightarrow \bar{*}$ & $h(\bar{*}) \rightarrow \bar{*}$ \\
\hline$\bullet \rightarrow *$ & $\bullet \rightarrow *$ & $\bullet \longrightarrow$ \\
\hline$a \rightarrow \mathrm{a}$ & $b \rightarrow \mathrm{b}$ & \\
\hline$b \rightarrow \mathrm{b}$ & $h(*) \rightarrow \overline{\mathrm{h}}(*)$ & \\
\hline$g(*) \rightarrow \mathrm{g}(*)$ & $h(\mathrm{~h}(* \overline{)}) \rightarrow \overline{\mathrm{h}(\mathrm{h}(*))}$ & \\
\hline$f(*, \mathrm{~b}) \rightarrow \mathrm{f}(*, \mathrm{~b})$ & & \\
\hline$f(\mathrm{~g}(*), \mathrm{a}) \rightarrow 1$ & $f(\mathrm{~h}(\mathrm{~h}(*)), *) \rightarrow 1$ & $* \rightarrow 1$ \\
\hline$h(\mathrm{a}) \rightarrow 2$ & $h(\overline{\mathrm{b}}) \rightarrow 2$ & $\bar{*} \rightarrow 2$ \\
\hline$h(\mathrm{f}(*, \mathrm{~b})) \rightarrow 3$ & $* \rightarrow 3$ & $* \rightarrow 3$ \\
\hline
\end{tabular}

\section{Shallow and Growing Approximations}

In this section we introduce the other two approximations that have been studied in the literature. The definitions below differ in a non-essential way from the corresponding ones in Comon [1] and Jacquemard [9].

Definition 19. A TRS $\mathcal{R}$ is called shallow if for every rewrite rule $l \rightarrow r \in \mathcal{R}$ the variables in $\mathcal{V} \operatorname{ar}(l) \cap \mathcal{V} \operatorname{ar}(r)$ occur at depth 1 in $l$ and at depth 0 or 1 in $r$. (The depth of a subterm occurrence is the length of its position.) We define $\mathcal{R}_{\text {sh }}$ as any right-linear shallow TRS that is obtained from $\mathcal{R}$ by replacing variables in the right-hand sides of the rewrite rules by variables that do not occur in the corresponding left-hand sides.

Definition 20. A TRS $\mathcal{R}$ is called growing if for every rewrite rule $l \rightarrow r \in \mathcal{R}$ the variables in $\mathcal{V} \operatorname{ar}(l) \cap \mathcal{V}$ ar $(r)$ occur at depth 1 in $l$. We define $\mathcal{R}_{\mathrm{g}}$ as any right-linear growing TRS that is obtained from $\mathcal{R}$ by replacing variables in the right-hand sides of the rewrite rules by variables that do not occur in the corresponding left-hand sides.

Clearly every right-ground TRS is shallow and every shallow TRS is growing. The reverse implications do not hold. Note that due to the right-linearity requirement $\mathcal{R}_{\text {sh }}$ and $\mathcal{R}_{\mathrm{g}}$ are not uniquely determined, even if we insist on replacing only variables that violate the restrictions imposed on shallow and growing TRSs. For instance, for $\mathcal{R}=\{f(x) \rightarrow g(x, x)\}$ we can take either one of $\mathcal{R}_{1}=\{f(x) \rightarrow g(y, x)\}, \mathcal{R}_{2}=\{f(x) \rightarrow g(x, y)\}$, and $\mathcal{R}_{3}=\{f(x) \rightarrow g(y, z)\}$ as $\mathcal{R}_{\text {sh }}$ (and $\mathcal{R}_{\mathrm{g}}$ ). Clearly $\mathcal{R}_{1}$ and $\mathcal{R}_{2}$ are better approximations of $\mathcal{R}$ than $\mathcal{R}_{3}$, 
but neither of $\mathcal{R}_{1}, \mathcal{R}_{2}$ approximates the other. All results stated below hold for any $\mathcal{R}_{\text {sh }}$ and $\mathcal{R}_{\mathrm{g}}$, provided of course we stick to our choices within a single 'environment'.

The proof of Lemma 17 doesn't extend to $\mathcal{R}_{\mathrm{sh}}$ and $\mathcal{R}_{\mathrm{g}}$ because the relations $\rightarrow_{\mathcal{R}_{\mathrm{sh}}}^{*}$ and $\rightarrow_{\mathcal{R}_{\mathrm{g}}}^{*}$ need not be recognizable. The following result is due to Comon [1] for $\mathcal{S}=\mathcal{R}_{\text {sh }}$ and Jacquemard [9] for $\mathcal{S}=\mathcal{R}_{\mathrm{g}}$.

Theorem 21 (Comon [1], Jacquemard [9]). Let $\mathcal{R}$ be a left-linear TRS. The set of terms that have a normal form with respect to $\mathcal{S}$ is recognizable for $\mathcal{S}=\mathcal{R}_{\mathrm{sh}}$ and $\mathcal{S}=\mathcal{R}_{\mathrm{g}}$.

The proof of Theorem 21 (in $[1,9]$ ) doesn't rely on the connection between weak second-order monadic logic and recognizability (i.e., Theorem 7), but is considerably more complicated than the proof of Lemma 16.

Lemma 22. Let $\mathcal{R}$ be a left-linear TRS. The set $\left(\rightarrow_{\mathcal{S}}^{*}\right)\left[\mathrm{NF}_{\mathcal{S}_{\mathbf{0}}}\right]$ is recognizable for $\mathcal{S}=\mathcal{R}_{\mathrm{sh}}$ and $\mathcal{S}=\mathcal{R}_{\mathrm{g}}$.

Proof. From $\rightarrow_{\mathcal{S}}^{*}=\rightarrow_{\mathcal{S}_{\text {. }}}^{*}$ it follows that $\left(\rightarrow_{\mathcal{S}}^{*}\right)\left[\mathrm{NF}_{\mathcal{S}_{\text {. }}}\right]$ coincides with the set of terms that have a normal form with respect to the TRS $\mathcal{S}_{\bullet}$. Since $\left(\mathcal{R}_{\mathrm{sh}}\right) \bullet$ $\left(\mathcal{R}_{\bullet}\right)_{\text {sh }}$ and $\left(\mathcal{R}_{\mathrm{g}}\right)_{\bullet}=\left(\mathcal{R}_{\bullet}\right)_{\mathrm{g}}$, the desired result follows from Theorem 21 .

Corollary 23. Let $\mathcal{R}$ be a left-linear TRS over a signature $\mathcal{F}$. It is decidable whether a redex in a term in $\mathcal{T}(\mathcal{F})$ is $\mathcal{S}$-needed for $\mathcal{S}=\mathcal{R}_{\mathrm{sh}}$ and $\mathcal{S}=\mathcal{R}_{\mathrm{g}}$.

It is easy to see that $\mathcal{R}_{\mathrm{s}}$-needed redexes in a term are always outermost. The same is true for $\mathcal{R}_{\mathrm{nv}}$-needed redexes in terms that have a normal form. However, $\mathcal{R}_{\mathrm{sh}}$-needed and $\mathcal{R}_{\mathrm{g}}$-needed redexes in normalizing terms need not be outermost. For instance, the TRS $\mathcal{R}=\{f(x) \rightarrow g(x), a \rightarrow b\}$ is shallow and hence $\mathcal{R}_{\text {sh }}=\mathcal{R}_{\mathrm{g}}=\mathcal{R}$. Innermost redex $a$ in the term $f(a)$ is $\mathcal{R}$-needed because there is no term $t \in \mathrm{NF}_{\mathcal{R}}$. such that $f(\bullet) \rightarrow_{\mathcal{R}}^{*} t$. Note that $a$ is not $\mathcal{R}_{\text {nv }}$-needed as $f(\bullet) \rightarrow_{\mathcal{R}_{\text {nv }}} g(b)$ with $g(b) \in \mathrm{NF}_{\mathcal{R}}$.

\section{Call by Need Computations to Normal Form}

A TRS $\mathcal{R}$ admits decidable call by need computations to normal form if it has an approximation $\mathcal{S}$ such that $\mathcal{S}$-needed redexes are computable and every term not in normal form has an $\mathcal{S}$-needed redex. This motivates the following definitions.

Definition 24. An approximation mapping is a mapping $\alpha$ from TRSs to TRSs with the property that $\alpha(\mathcal{R})$ approximates $\mathcal{R}$, for every TRS $\mathcal{R}$. In the following we write $\mathcal{R}_{\alpha}$ instead of $\alpha(\mathcal{R})$. We define a partial order $\leqslant$ on approximation mappings as follows: $\alpha \leqslant \beta$ if and only if $\mathcal{R}_{\beta}$ approximates $\mathcal{R}_{\alpha}$, for every TRS $\mathcal{R}$. Note that the identity mapping is the minimum element of this partial order.

The mapping $\mathrm{s}$ that assigns to every TRS $\mathcal{R}$ the TRS $\mathcal{R}_{\mathrm{s}}$ is an approximation mapping. In the same fashion, Definitions 13, 19, and 20 define approximation mappings nv, sh, and $\mathrm{g}$. We have $\mathrm{g} \leqslant \mathrm{sh} \leqslant \mathrm{nv} \leqslant \mathrm{s}$. 
Definition 25. Let $\alpha$ be an approximation mapping. The class of TRSs $\mathcal{R}$ such that every reducible term in $\mathcal{T}(\mathcal{F})$ has an $\mathcal{R}_{\alpha}$-needed redex is denoted by $\mathrm{CBN}-\mathrm{NF}_{\alpha}$. Here $\mathcal{F}$ denotes the signature of $\mathcal{R}$.

Lemma 26. Let $\mathcal{R}$ be an orthogonal TRS. If $\mathcal{R}$ is right-ground then $\mathcal{R} \in \mathrm{CBN}^{-\mathrm{NF}_{\mathrm{nv}}}$.

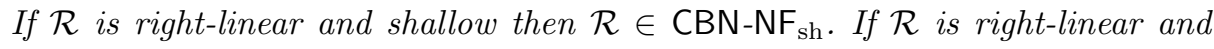
growing then $\mathcal{R} \in \mathrm{CBN}-\mathrm{NF}_{\mathrm{g}}$.

Proof. According to Theorem 1 and the remark following Definition 10 every reducible term contains an $\mathcal{R}$-needed redex. If $\mathcal{R}$ is right-ground then $\mathcal{R}=\mathcal{R}_{\mathrm{nv}}$ and thus $\mathcal{R} \in \mathrm{CBN}^{-N F_{\text {nv }}}$. If $\mathcal{R}$ is right-linear and shallow then $\mathcal{R}=\mathcal{R}_{\text {sh }}$ and

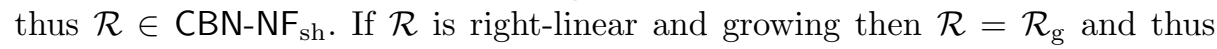
$\mathcal{R} \in \mathrm{CBN}-\mathrm{NF}_{\mathrm{g}}$.

The next lemma is an easy consequence of Lemma 11.

Lemma 27. Let $\alpha$ and $\beta$ be approximation mappings. If $\alpha \leqslant \beta$ then $\mathrm{CBN}^{-\mathrm{NF}_{\beta}} \subseteq$ $\mathrm{CBN}-\mathrm{NF}_{\alpha}$.

In this section we prove that membership of CBN-NF ${ }_{\alpha}$ for $\alpha \in\{\mathrm{s}, \mathrm{nv}, \mathrm{sh}, \mathrm{g}\}$ is decidable for left-linear TRSs. The proof is a straightforward consequence of the following result.

Theorem 28. Let $\mathcal{R}$ be a left-linear TRS such that $\mathcal{R}$-NEEDED is recognizable. The set of terms that have an $\mathcal{R}$-needed redex is recognizable.

Proof. Let $\mathcal{F}$ be the signature of $\mathcal{R}$. Define the relation $\operatorname{mark}_{\mathcal{R}}^{\bullet}$ on $\mathcal{T}\left(\mathcal{F}_{\bullet}\right)$ as the parallel closure of $\{(\Delta, \bullet) \mid \Delta \in \mathcal{T}(\mathcal{F})$ is a redex $\}$. The set of terms that have an $\mathcal{R}$-needed redex coincides with $\operatorname{mark}_{\mathcal{R}}^{\bullet}[\mathcal{R}$-NEEDED $] \cap \mathcal{T}(\mathcal{F})$. If we can show that the relation mark $_{\mathcal{R}}^{\bullet}$ is recognizable then the result follows from Lemmata 3 and 6 . Let $\mathcal{A}$ be a tree automaton with a unique final state ! that accepts $\operatorname{REDEX}_{\mathcal{R}} \cap \mathcal{T}(\mathcal{F})$ and let $\mathcal{B}$ be the tree automaton whose single transition rule is $\bullet \rightarrow$ !. It is not difficult to see that the $\operatorname{GTT}(\mathcal{A}, \mathcal{B})$ accepts mark ${ }_{\mathcal{R}}^{\bullet}$.

Theorem 29. Let $\mathcal{R}$ be a left-linear TRS and let $\alpha$ be an approximation mapping such that $\mathcal{R}_{\alpha}$-NEEDED is recognizable. It is decidable whether $\mathcal{R} \in \mathrm{CBN}-\mathrm{NF}_{\alpha}$.

Proof. Let $\mathcal{F}$ be the signature of $\mathcal{R}$. The TRS $\mathcal{R}$ belongs to $\mathrm{CBN}^{-N F_{\alpha}}$ if and only if the set

$$
A=\left(\mathcal{T}(\mathcal{F}) \backslash \mathrm{NF}_{\mathcal{R}}\right) \backslash\left\{t \in \mathcal{T}(\mathcal{F}) \mid t \text { has an } \mathcal{R}_{\alpha} \text {-needed redex }\right\}
$$

is empty. Because $A$ is recognizable due to Theorem 28 and Lemmata 3 and 5 , emptiness of $A$ is decidable by the second part of Lemma 4 .

Corollary 30. Let $\mathcal{R}$ be a left-linear TRS and let $\alpha \in\{\mathrm{s}, \mathrm{nv}, \mathrm{sh}, \mathrm{g}\}$. It is decidable whether $\mathcal{R} \in \mathrm{CBN}-\mathrm{NF}_{\alpha}$.

It shouldn't come as a surprise that a better approximation covers a larger class of TRSs. This is expressed formally in the next lemma. 
Lemma 31. We have $\mathrm{CBN}-\mathrm{NF}_{\mathrm{s}} \subset \mathrm{CBN}-\mathrm{NF}_{\mathrm{nv}} \subset \mathrm{CBN}-\mathrm{NF}_{\mathrm{sh}} \subset \mathrm{CBN}-\mathrm{NF}_{\mathrm{g}}$, even when these classes are restricted to orthogonal TRSs.

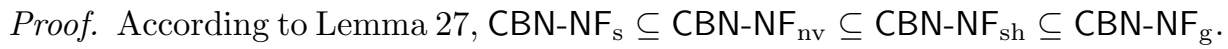
Consider the orthogonal TRSs

$$
\begin{array}{rll}
\mathcal{R}_{1} & \multicolumn{1}{c}{\mathcal{R}_{2}} & \multicolumn{1}{c}{\mathcal{R}_{3}} \\
f(a, b, x) \rightarrow a & f(a, b, x) \rightarrow a & f(a, b, x) \rightarrow a \\
f(b, x, a) \rightarrow b & f(b, x, a) \rightarrow b & f(b, x, a) \rightarrow b \\
f(x, a, b) \rightarrow c & f(x, a, b) \rightarrow x & f(x, a, b) \rightarrow g(g(x)) \\
& & g(x) \rightarrow x
\end{array}
$$

According to Lemma $26 \mathcal{R}_{1} \in \mathrm{CBN}-\mathrm{NF}_{\mathrm{nv}}, \mathcal{R}_{2} \in \mathrm{CBN}-\mathrm{NF}_{\mathrm{sh}}$, and $\mathcal{R}_{3} \in \mathrm{CBN}-\mathrm{NF}_{\mathrm{g}}$. So it remains to show that $\mathcal{R}_{1} \notin \mathrm{CBN}-\mathrm{NF}_{\mathrm{s}}, \mathcal{R}_{2} \notin \mathrm{CBN}-\mathrm{NF}_{\mathrm{nv}}$, and $\mathcal{R}_{3} \notin \mathrm{CBN}-\mathrm{NF}$ sh. We have

$$
\begin{array}{rrl}
\left(\mathcal{R}_{1}\right)_{\mathrm{s}} & \left(\mathcal{R}_{2}\right)_{\mathrm{nv}} & \multicolumn{1}{c}{\left(\mathcal{R}_{3}\right)_{\mathrm{sh}}} \\
f(a, b, x) \rightarrow y & f(a, b, x) \rightarrow a & f(a, b, x) \rightarrow a \\
f(b, x, a) \rightarrow y & f(b, x, a) \rightarrow b & f(b, x, a) \rightarrow b \\
f(x, a, b) \rightarrow y & f(x, a, b) \rightarrow y & f(x, a, b) \rightarrow g(g(y))
\end{array}
$$

Let $\Delta$ be the redex $f(a, a, b)$. The following rewrite sequences in $\left(\mathcal{R}_{1}\right)_{\mathrm{s}}$ show that none of the redexes in $f(\Delta, \Delta, \Delta)$ is $\left(\mathcal{R}_{1}\right)_{\mathrm{s}}$-needed: $f(\bullet, \Delta, \Delta) \rightarrow f(\bullet, a, \Delta) \rightarrow$ $f(\bullet, a, b) \rightarrow a, f(\Delta, \bullet, \Delta) \rightarrow f(b, \bullet, \Delta) \rightarrow f(b, \bullet, a) \rightarrow a, f(\Delta, \Delta, \bullet) \rightarrow f(a, \Delta, \bullet)$ $\rightarrow f(a, b, \bullet) \rightarrow a$. Hence $\mathcal{R}_{1} \notin \mathrm{CBN}-\mathrm{NF}_{\mathrm{s}}$. In $\left(\mathcal{R}_{2}\right)_{\mathrm{nv}}$ we have $\Delta \rightarrow t$ for every term $t$ and thus $f(\bullet, \Delta, \Delta) \rightarrow f(\bullet, a, \Delta) \rightarrow f(\bullet, a, b) \rightarrow a, f(\Delta, \bullet, \Delta) \rightarrow f(b, \bullet, \Delta) \rightarrow$ $f(b, \bullet, a) \rightarrow b$, and $f(\Delta, \Delta, \bullet) \rightarrow f(a, \Delta, \bullet) \rightarrow f(a, b, \bullet) \rightarrow a$. Consequently,

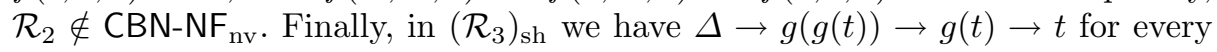
term $t$. Hence $f(\bullet, \Delta, \Delta) \rightarrow^{*} f(\bullet, a, \Delta) \rightarrow^{*} f(\bullet, a, b) \rightarrow g(g(a)) \rightarrow g(a) \rightarrow$ $a, f(\Delta, \bullet, \Delta) \rightarrow^{*} f(b, \bullet, \Delta) \rightarrow^{*} f(b, \bullet, a) \rightarrow b, f(\Delta, \Delta, \bullet) \rightarrow^{*} f(a, \Delta, \bullet) \rightarrow^{*}$ $f(a, b, \bullet) \rightarrow a$, and therefore $\mathcal{R}_{3} \notin \mathrm{CBN}-\mathrm{NF}_{\mathrm{g}}$.

It can be shown that $\mathrm{CBN}_{\mathrm{N}} \mathrm{NF}_{\mathrm{s}}$ coincides with the class of strongly sequential TRSs introduced by Huet and Lévy [8]. However, $\mathrm{CBN}^{-N F_{n v}}$ properly includes the class of NV-sequential TRSs introduced by Oyamaguchi [16] as well as the extension to NVNF-sequential TRSs considered by Nagaya et al. [15]. For instance, the TRS $\mathcal{R}_{1}$ defined in the above proof is neither NV-sequential nor NVNFsequential (because the term $f(\Omega, \Omega, \Omega)$ doesn't have an index). Figure 1 shows the relationship between several classes of TRSs that admit decidable call by need computations to normal form. Areas (1), (2), (3), and (4) consist of all NV, NVNF, shallow (Comon [1]), and growing (Jacquemard [9]) sequential TRSs, respectively. Note that $C B N-N F_{s h}$ and $C B N-N F_{g}$ properly include the classes of shallow and growing sequential TRSs.

Why do we get more powerful results with so little effort? The deeper reason is that needed redexes are not independent of other redexes in the same term. Consider for instance the redexes $\Delta_{1}=f(a, b, a)$ and $\Delta_{2}=f(b, a, b)$ with respect to the TRS $\mathcal{R}_{1}$ in the proof of Lemma 31 . An arbitrary redex $\Delta$ is needed in the 


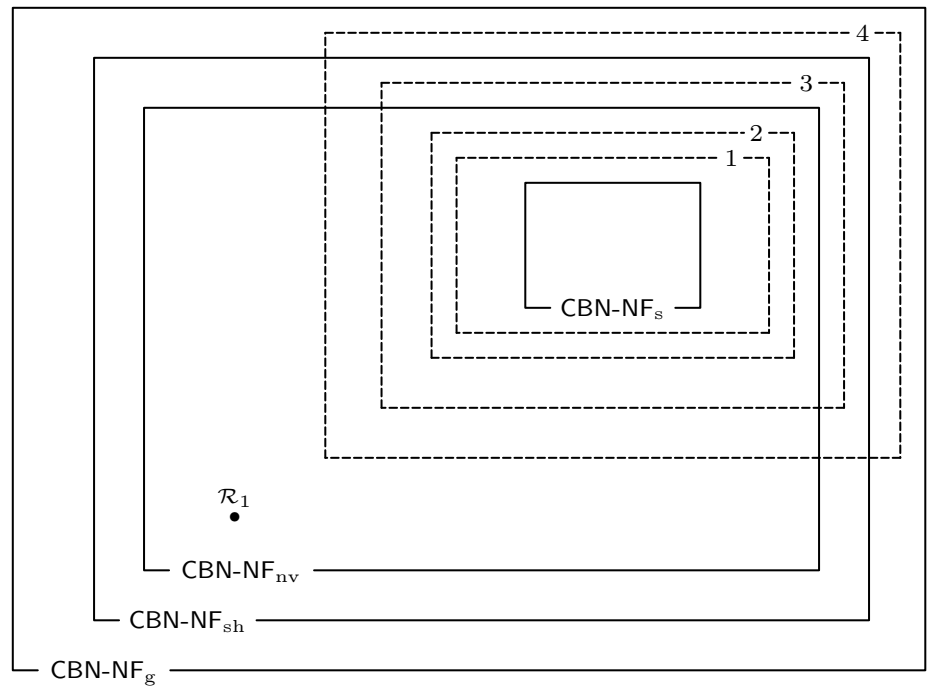

Fig. 1. Comparison.

term $f\left(\Delta_{1}, \Delta_{1}, \Delta\right)$ but not in the term $f\left(\Delta_{1}, \Delta_{2}, \Delta\right)$. The non-independence of needed redexes implies that if all (outermost) redexes in a term are replaced by a new constant $\Omega$, which happens in the traditional approach to decidable approximations to call by need based on the notions of index and sequentiality, essential information for determining needed redexes is lost. Only when considering the approximation mapping s the use of $\Omega$ entails no loss of generality because in $\mathcal{R}_{\mathrm{s}}$ redexes reduce to all possible terms. This is the reason why $\mathrm{CBN}-\mathrm{NF}_{\mathrm{s}}$ coincides with the class of strongly sequential TRSs.

\section{Decidable Approximations of Root-Neededness}

In the remainder of this paper we generalize the results of the preceding sections to call by need computations to root-stable forms. The first problem we face is to find a definition of root-needed redex that doesn't depend on the notion of descendant. This is less trivial than it seems, because the obvious adaptation

redex $\Delta$ in term $C[\Delta] \in \mathcal{T}(\mathcal{F})$ is root-needed if and only if there is no term $t \in \mathrm{RS}_{\mathcal{R}}$ such that $C[\bullet] \rightarrow_{\mathcal{R}}^{*} t$

of Lemma 8 doesn't work. For instance, redex $a$ in the term $f(a)$ is root-needed with respect to the TRS $\mathcal{R}=\{a \rightarrow b, f(b) \rightarrow c\}$ but $f(\bullet)$ is root-stable with respect to $\mathcal{R}_{\boldsymbol{e}}$. On the other hand, redex $f(b)$ in the term $f(f(b))$ is not rootneeded as $f(f(b))$ is root-stable. This shows that root-needed redexes, unlike needed redexes, are not uniform: root-neededness of a redex doesn't depend only on its position in a term. Hence when trying to determine whether a redex $\Delta$ is root-needed we cannot simply replace it by $\bullet$. Instead, we mark the root symbol of $\Delta$. 
Definition 32. Let $\mathcal{F}$ be a signature. Let $\mathcal{F}_{\circ}=\mathcal{F} \cup\left\{f_{\circ} \mid f \in \mathcal{F}\right\}$ where every $f_{\circ}$ has the same arity as $f$. Let $\mathcal{R}$ be a TRS over the signature $\mathcal{F}$. Let $\Delta \in \mathcal{T}(\mathcal{F})$ be a redex. We write $\Delta^{\circ}$ for the term that is obtained from $\Delta$ by marking its root symbol, i.e., if $\Delta=f\left(t_{1}, \ldots, t_{n}\right)$ then $\Delta^{\circ}=f_{\circ}\left(t_{1}, \ldots, t_{n}\right)$. We call $\Delta^{\circ}$ a marked redex. The mapping from $\mathcal{T}\left(\mathcal{F}_{\circ}\right)$ to $\mathcal{T}(\mathcal{F})$ that simply erases all marks is denoted by erase, so erase $\left(f\left(t_{1}, \ldots, t_{n}\right)\right)=\operatorname{erase}\left(f_{\circ}\left(t_{1}, \ldots, t_{n}\right)\right)=f\left(\operatorname{erase}\left(t_{1}\right), \ldots, \operatorname{erase}\left(t_{n}\right)\right)$.

The marking of redexes serves a double purpose. On the one hand, it tells us which redex we want to test for root-neededness. On the other hand, marked redexes are not redexes - simply because there are no marks in the (left-hand sides of the) rewrite rules - so if we rewrite a term that contains a marked redex, the marked redex is never contracted.

Lemma 33. Let $\mathcal{R}$ be an orthogonal TRS over a signature $\mathcal{F}$. Redex $\Delta$ in term $C[\Delta] \in \mathcal{T}(\mathcal{F})$ is root-needed if and only if there is no term $t$ such that $C\left[\Delta^{\circ}\right] \rightarrow_{\mathcal{R}}^{*} t$ and $\operatorname{erase}(t) \in \mathrm{RS}_{\mathcal{R}}$.

(Due to lack of space, we omit all proofs in this section.) Actually, we can do without the relation erase because for orthogonal TRSs $\mathcal{R}$ it is not difficult to prove that $\left\{t \in \mathcal{T}\left(\mathcal{F}_{\circ}\right) \mid\right.$ erase $\left.(t) \in \mathrm{RS}_{\mathcal{R}}\right\}$ coincides with $\mathrm{RS}_{\mathcal{R}}$ where $\mathcal{R}_{\circ}$ denotes the TRS $\mathcal{R} \cup\left\{l^{\circ} \rightarrow r \mid l \rightarrow r \in \mathcal{R}\right\}$. Hence redex $\Delta$ in term $C[\Delta] \in \mathcal{T}(\mathcal{F})$ is root-needed if and only if there is no term $t \in \mathrm{RS}_{\mathcal{R}_{\circ}}$ such that $C\left[\Delta^{\circ}\right] \rightarrow_{\mathcal{R}}^{*} t$.

Besides the non-computability of $\rightarrow_{\mathcal{R}}^{*}$ we face the additional problem that root-stability is in general not computable. So in order to arrive at decidable approximations of root-neededness, we approximate $\mathcal{R}$ by TRSs $\mathcal{S}_{1}$ and $\mathcal{S}_{2}$ such that it is decidable whether a term has an $\mathcal{S}_{1}$-reduct in $\operatorname{RS}_{\left(\mathcal{S}_{2}\right)_{0}}$.

Definition 34. Let $\mathcal{S}_{1}$ and $\mathcal{S}_{2}$ be TRSs over the same signature $\mathcal{F}$. We say that redex $\Delta$ in $C[\Delta] \in \mathcal{T}(\mathcal{F})$ is $\left(\mathcal{S}_{1}, \mathcal{S}_{2}\right)$-root-needed if there is no term $t \in \mathrm{RS}_{\left(\mathcal{S}_{2}\right)}$ 。 such that $C\left[\Delta^{\circ}\right] \rightarrow_{\mathcal{S}_{1}}^{*} t$. We abbreviate $(\mathcal{S}, \mathcal{S})$-root-needed to $\mathcal{S}$-root-needed.

Our $\mathcal{R}_{\mathrm{s}}$-root-needed redexes coincide with the strongly root-needed redexes of Kennaway [11].

Lemma 35. Let $\mathcal{R}_{1}$ and $\mathcal{R}_{2}$ be TRSs over the same signature with approximations $\mathcal{S}_{1}$ and $\mathcal{S}_{2}$. Every $\left(\mathcal{S}_{1}, \mathcal{R}_{2}\right)$-root-needed redex is $\left(\mathcal{R}_{1}, \mathcal{S}_{2}\right)$-root-needed.

It should be noted that, for an approximation $\mathcal{S}$ of $\mathcal{R}, \mathcal{S}$-root-needed redexes need not be $\mathcal{R}$-root-needed. Consider for instance the orthogonal TRS $\mathcal{R}=$ $\{a \rightarrow b, f(c) \rightarrow c\}$. The term $f(a)$ is root-stable, hence its redex $a$ is not rootneeded. Nevertheless, redex $a$ is $\mathcal{R}_{\mathrm{s}}$-needed because the only term $t$ such that $f\left(a_{\circ}\right) \rightarrow{ }_{\mathcal{R}_{\mathrm{s}}}^{*} t$ is $f\left(a_{\circ}\right)$ itself and $f\left(a_{\circ}\right) \notin \mathrm{RS}_{\left(\mathcal{R}_{\mathrm{s}}\right)_{\circ}}$ because we have $f\left(a_{\circ}\right) \rightarrow f(c)$ in the TRS $\left(\mathcal{R}_{\mathrm{s}}\right)_{\circ}=\left\{a \rightarrow x, a_{\circ} \rightarrow x, f(c) \rightarrow x, f_{\circ}(c) \rightarrow x\right\}$. So not every strongly root-needed redex is root-needed, contradicting Theorem 16 in [11].

Definition 36. Let $\mathcal{R}$ and $\mathcal{S}$ be TRSs over the same signature $\mathcal{F}$. The set of all terms $C\left[\Delta^{\circ}\right] \in \mathrm{M}_{\mathcal{R}}^{\circ}$ such that there is no term $t \in \mathrm{RS}_{\mathcal{S}_{\circ}}$ with $C\left[\Delta^{\circ}\right] \rightarrow_{\mathcal{R}}^{*} t$ is denoted by $(\mathcal{R}, \mathcal{S})$-ROOT-NEEDED. Here $\mathrm{M}_{\mathcal{R}}^{\circ}=\left\{C\left[\Delta^{\circ}\right] \mid C[\Delta] \in \mathcal{T}(\mathcal{F})\right.$ and $\Delta$ is an $\mathcal{R}$-redex $\}$ consists of all terms that contain exactly one marked redex. 
Theorem 37. Let $\mathcal{R}$ and $\mathcal{S}$ be TRSs over the same signature $\mathcal{F}$. If $\left(\rightarrow \rightarrow_{\mathcal{R}}^{*}\right)\left[\mathrm{RS}_{\mathcal{S}_{0}}\right]$ is recognizable and $\mathcal{R}$ left-linear then $(\mathcal{R}, \mathcal{S})$-ROOT-NEEDED is recognizable.

The following lemma states that the first premise of Theorem 37 is satisfied for the four approximations defined in the preceding two sections.

Lemma 38. Let $\mathcal{R}$ be a left-linear TRS. The set $\left(\rightarrow_{\mathcal{R}_{\alpha}}^{*}\right)\left[\mathrm{RS}_{\left(\mathcal{R}_{\beta}\right)_{0}}\right]$ is recognizable for $\alpha, \beta \in\{\mathrm{s}, \mathrm{nv}, \mathrm{sh}, \mathrm{g}\}$.

Corollary 39. Let $\mathcal{R}$ be a left-linear TRS over a signature $\mathcal{F}$. It is decidable whether a redex in a term in $\mathcal{T}(\mathcal{F})$ is $\left(\mathcal{R}_{\alpha}, \mathcal{R}_{\beta}\right)$-root-needed for $\alpha, \beta \in$ $\{\mathrm{s}, \mathrm{nv}, \mathrm{sh}, \mathrm{g}\}$.

\section{Call by Need Computations to Root-Stable Form}

Definition 40. Let $\alpha$ and $\beta$ be approximation mappings. The class of TRSs $\mathcal{R}$ such that every non- $\mathcal{R}_{\beta}$-root-stable term in $\mathcal{T}(\mathcal{F})$ has an $\left(\mathcal{R}_{\alpha}, \mathcal{R}_{\beta}\right)$-root-needed redex is denoted by $\mathrm{CBN}-\mathrm{RS}_{\alpha, \beta}$. Here $\mathcal{F}$ denotes the signature of $\mathcal{R}$.

It can be shown that $C B N-R_{s, s}$ coincides with the class of strongly rootsequential TRSs introduced by Kennaway [11].

The following result corresponds to Theorem 28 . The proof, however, is more difficult because the parallel closure of $\left\{\left(\Delta, \Delta^{\circ}\right) \mid \Delta \in \mathcal{T}(\mathcal{F})\right.$ is a redex $\}$ is not recognizable since the size of redexes is unbounded and in GTTs one can only transfer a finite amount of information between the two sides. We overcome this problem by resorting to weak second-order monadic logic.

Theorem 41. Let $\mathcal{R}$ and $\mathcal{S}$ be TRSs such that $(\mathcal{R}, \mathcal{S})$-ROOT-NEEDED is recognizable. The set of terms that have an $(\mathcal{R}, \mathcal{S})$-root-needed redex is recognizable.

Proof. Let $\phi(\mathbf{X})$ be the definition of $(\mathcal{R}, \mathcal{S})$-ROOT-NEEDED in WS $k$ S, whose existence is guaranteed by Theorem 7 . The set of terms that have an $(\mathcal{R}, \mathcal{S})$ root-needed redex is defined by the following WS $k \mathrm{~S}$ formula:

$$
\overbrace{\bigwedge_{f \in \mathcal{F}_{\circ} \backslash \mathcal{F}} X_{f}=\varnothing}^{(1)} \wedge \exists \mathbf{Y}[\overbrace{X=Y \wedge \bigwedge_{f \in \mathcal{F}} X_{f}=Y_{f} \cup Y_{f_{\circ}}}^{(2)} \wedge \overbrace{\phi(\mathbf{Y})}^{(3)}] .
$$

Part (1) ensures that the term $t$ encoded by $\mathbf{X}$ contains no marks, part (2) ensures that erase $\left(t^{\prime}\right)=t$ for the term $t^{\prime}$ encoded by $\mathbf{Y}$, and part (3) ensures that $t^{\prime} \in(\mathcal{R}, \mathcal{S})$-ROOT-NEEDED. Hence the result follows from Theorem 7 .

Theorem 42. Let $\mathcal{R}$ be a TRS and let $\alpha$ and $\beta$ be approximation mapping such that $\mathrm{RS}_{\mathcal{R}_{\beta}}$ and $\left(\mathcal{R}_{\alpha}, \mathcal{R}_{\beta}\right)$-ROOT-NEEDED are recognizable. It is decidable whether $\mathcal{R} \in \mathrm{CBN}-\mathrm{RS}_{\alpha, \beta}$. 
Proof. Let $\mathcal{F}$ be the signature of $\mathcal{R}$. The TRS $\mathcal{R}$ belongs to $\mathrm{CBN}^{-\mathrm{RS}_{\alpha, \beta}}$ if and only if the set

$$
A=\left(\mathcal{T}(\mathcal{F}) \backslash \mathrm{RS}_{\mathcal{R}_{\beta}}\right) \backslash\left\{t \in \mathcal{T}(\mathcal{F}) \mid t \text { has an }\left(\mathcal{R}_{\alpha}, \mathcal{R}_{\beta}\right) \text {-root-needed redex }\right\}
$$

is empty. Because $A$ is recognizable due to Theorem 41 and Lemma 3, emptiness of $A$ is decidable by the second part of Lemma 4 .

\section{References}

1. H. Comon, Sequentiality, Second-Order Monadic Logic and Tree Automata, Proc. 10th LICS (1995) 508-517.

2. J.L. Coquidé and R. Gilleron, Proofs and Reachability Problem for Ground Rewrite Systems, Proc. 6th International Meeting of Young Computer Scientists, LNCS 464 (1990) 120-129.

3. M. Dauchet, T. Heuillard, P. Lescanne, and S. Tison, Decidability of the Confluence of Finite Ground Term Rewriting Systems and of Other Related Term Rewriting Systems, IC 88 (1990) 187-201.

4. M. Dauchet and S. Tison, Decidability of Confluence for Ground Term Rewriting Systems, Proc. 1st FCT, LNCS 199 (1985) 80-89.

5. N. Dershowitz and J.-P. Jouannaud, Rewrite Systems, in: Handbook of Theoretical Computer Science, Vol. B, North-Holland (1990) 243-320.

6. J. Doner, Tree Acceptors and Some of Their Applications, JCSS 4 (1970) 406-451.

7. F. Gécseg and M. Steinby, Tree Automata, Akadémiai Kiadó, Budapest, 1984.

8. G. Huet and J.-J. Lévy, Computations in Orthogonal Rewriting Systems, I and II, in: Computational Logic, Essays in Honor of Alan Robinson, The MIT Press (1991) 396-443.

9. F. Jacquemard, Decidable Approximations of Term Rewriting Systems, Proc. 7th RTA, LNCS 1103 (1996) 362-376.

10. J.-P. Jouannaud and W. Sadfi, Strong Sequentiality of Left-Linear Overlapping Rewrite Systems, Proc. 4th CTRS, LNCS 968 (1995) 235-246.

11. J.R. Kennaway, A Conflict Between Call-by-Need Computation and Parallelism, Proc. 4th CTRS, LNCS 968 (1995) 247-261.

12. J.W. Klop, Term Rewriting Systems, in: Handbook of Logic in Computer Science, Vol. 2, Oxford University Press (1992) 1-116.

13. J.W. Klop and A. Middeldorp, Sequentiality in Orthogonal Term Rewriting Systems, JSC 12 (1991) 161-195.

14. A. Middeldorp, Call by Need Computations to Root-Stable Form, Proc. 24th POPL (1997) 94-105.

15. T. Nagaya, M. Sakai, and Y. Toyama, NVNF-Sequentiality of Left-Linear Term Rewriting Systems, Proc. RIMS Workshop on Theory and Applications of Rewriting, RIMS Technical Report 918, University of Kyoto (1995) 109-117.

16. M. Oyamaguchi, NV-Sequentiality: A Decidable Condition for Call-by-Need Computations in Term Rewriting Systems, SIAM J. on Comput. 22 (1993) 114-135.

17. J.W. Thatcher and J.B. Wright, Generalized Finite Automata Theory with an Application to a Decision Problem of Second-Order Logic, MST 2 (1968) 57-81.

18. W. Thomas, Automata on Infinite Objects, in: Handbook of Theoretical Computer Science, Vol. B, North-Holland (1990) 133-191.

19. Y. Toyama, Strong Sequentiality of Left-Linear Overlapping Term Rewriting Systems, Proc. 7th LICS (1992) 274-284. 